\title{
Interactions of the $\beta$-blocker drug, propranolol, with detergents, $\beta$-cyclodextrin and living cells studied using fluorescence spectroscopy and imaging
}

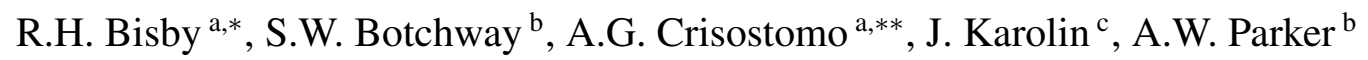 \\ and L. Schröder ${ }^{\text {a }}$ \\ ${ }^{a}$ School of Environment and Life Sciences, Peel Building, University of Salford, Salford, UK \\ ${ }^{\mathrm{b}}$ Lasers for Science Facility, Central Laser Facility, Science and Technology Facilities Council, \\ Rutherford Appleton Laboratory, Harwell Science and Innovation Campus, Didcot, UK \\ ${ }^{\mathrm{c}}$ Department of Physics, SUPA, University of Strathclyde, Glasgow, UK
}

\begin{abstract}
Interactions of the $\beta$-blocker drug, propranolol, with amphipathic systems have been studied using fluorescence spectroscopy. The results show a strong binding of propranolol with micelles of sodium dodecyl sulfate revealed through changes in the fluorescence spectrum and an increase in fluorescence lifetime. Quenching of propranolol fluorescence by iodide is used to demonstrate interaction with $\beta$-cyclodextrin. At high concentrations, self-quenching of propranolol fluorescence was also observed with $k_{\mathrm{q}}=2.5 \times 10^{9} \mathrm{dm}^{3} \mathrm{~mol}^{-1} \mathrm{~s}^{-1}$. Two-photon excited $(630 \mathrm{~nm})$ fluorescence lifetime imaging of propranolol in cells showed propranolol to be widely distributed in the cell cytoplasm, with fluorescence lifetimes shorter than in solution. The results suggest that intracellular propranolol is mainly confined within the aqueous cytoplasm and rather than membrane associated.
\end{abstract}

Keywords: Propranolol, fluorescence, lifetime, quenching, micelle, cyclodextrin, imaging, live cell

\section{Introduction}

Propranolol (I) is a drug used to regulate blood pressure and anxiety levels [7,12]. As a $\beta$-blocker it prevents binding of epinephrine and norepinephrine to cell surface receptors and inhibits intracellular signalling cascades. Propranolol is an amphipathic molecule that interacts with cellular membranes [18]. Binding of propranolol to lipid bilayers is stronger association for the neutral species in alkaline solution than for the protonated form at neutral $\mathrm{pH}$ [1]. In contrast the evident hydrophilic character of propranolol is demonstrated by its high solubility in neutral aqueous solutions (up to $50 \mathrm{mmol} \mathrm{dm}{ }^{-3}$ ). Recent studies [10] have shown that propranolol is rapidly taken up by live mammalian cells, but the intracellular location of accumulated propranolol was unknown. With convenient absorption and fluorescent

\footnotetext{
${ }^{*}$ Corresponding author: Prof. R.H. Bisby, School of Environment and Life Sciences, Peel Building, University of Salford, Salford M5 4WT, UK. Tel.: +44 161295 4912; Fax: +44 161295 5015; E-mail: r.h.bisby@ salford.ac.uk.

*** Present address: MRC Laboratory of Molecular Biology, Hills Road, Cambridge, CB2 0QH, UK.
} 
properties enabling it to be selectively excited in cellular environments $[3,4]$, intracellular propranolol may be imaged during uptake into live mammalian cells.

\section{Materials and methods}

$( \pm$ )-Propranolol hydrochloride and other chemicals were obtained from Sigma-Aldrich. Fluorescence spectra were recorded using a Spex Fluoromax fluorimeter and absorption spectra were measured with a Perkin-Elmer Lambda-25 spectrometer. Fluorescence lifetimes with one-photon excitation were determined by time-correlated single photon counting (TCSPC) apparatus (IBH with pulsed LED excitation at $296 \mathrm{~nm})$.

\section{Results and discussion}

As previously observed $[11,18]$, the fluorescence spectra of propranolol vary with solvent. In ethanol (Fig. 1), methanol and acetonitrile a structured emission with peaks at 321, 336 and $352 \mathrm{~nm}$ is observed. In water, dimethylformamide and dimethyl sulfoxide an additional broad band at longer wavelength at $370-380 \mathrm{~nm}$ is observed. The spectrum in neutral aqueous buffer was invariant with concentration in the micromolar region, excluding excimer as the origin of this additional band. There is no obvious correlation of intensities at 336 and $380 \mathrm{~nm}$ with solvent dielectric constant and the additional band observed in water, DMF and DMSO is suggested to stem from charge transfer between the naphthalene ring and the side chain depending on strong hydrogen bond accepting character of the solvent.

Fluorescence spectra recorded on titration of propranolol with sodium dodecyl sulfate (SDS) show (Fig. 1) a distinct change at the critical micelle concentration (CMC, ca $1 \mathrm{mmol} \mathrm{dm}^{-3}$ ) although more subtle changes are also apparent pre-micellization. Below the CMC spectra are similar to that in water. Above the CMC spectra display the structure observed in solvents such as ethanol and lack the charge transfer band. This suggests association between propranolol and the micelle with the naphthalene ring residing within the micelle and shielded from the aqueous solvent. Similar experiments with neutral (reduced Triton X-100) and cationic (cetyltrimethyl ammonium chloride) micelles failed to show similar interactions and indicate the important contribution from the anionic head group of SDS in the binding process. Changes in fluorescence spectra (Fig. 1) during titration of propranolol with SDS and the solvent effects are very much more pronounced than for naphthalene itself [14,15]. This provides strong support for the assignment of the long wavelength band in solvents such as water and DMSO to an intramolecular charge transfer state as previously described in related molecules [5,17].

\section{Fluorescence lifetimes}

Fluorescence lifetimes of propranolol were measured using one-photon excitation at $296 \mathrm{~nm}$. Lifetimes in aqueous solutions measured with two-photon excitation at $630 \mathrm{~nm}$ gave essentially identical results. In neutral aqueous solutions the fluorescence decay gave a good single exponential fit with a lifetime of $10.0 \mathrm{~ns}$. At $\mathrm{pH} 11$, above the $p K_{\mathrm{a}}$ of the secondary amine function $\left(p K_{\mathrm{a}}=9.5\right)$ the lifetime was $6.55 \mathrm{~ns}$. In non-aqueous solvents the fluorescence decay was biexponential. In deaerated ethanol the dominant longer lifetime component was $14.8 \mathrm{~ns}$, whereas in aerated DMSO the dominant component 


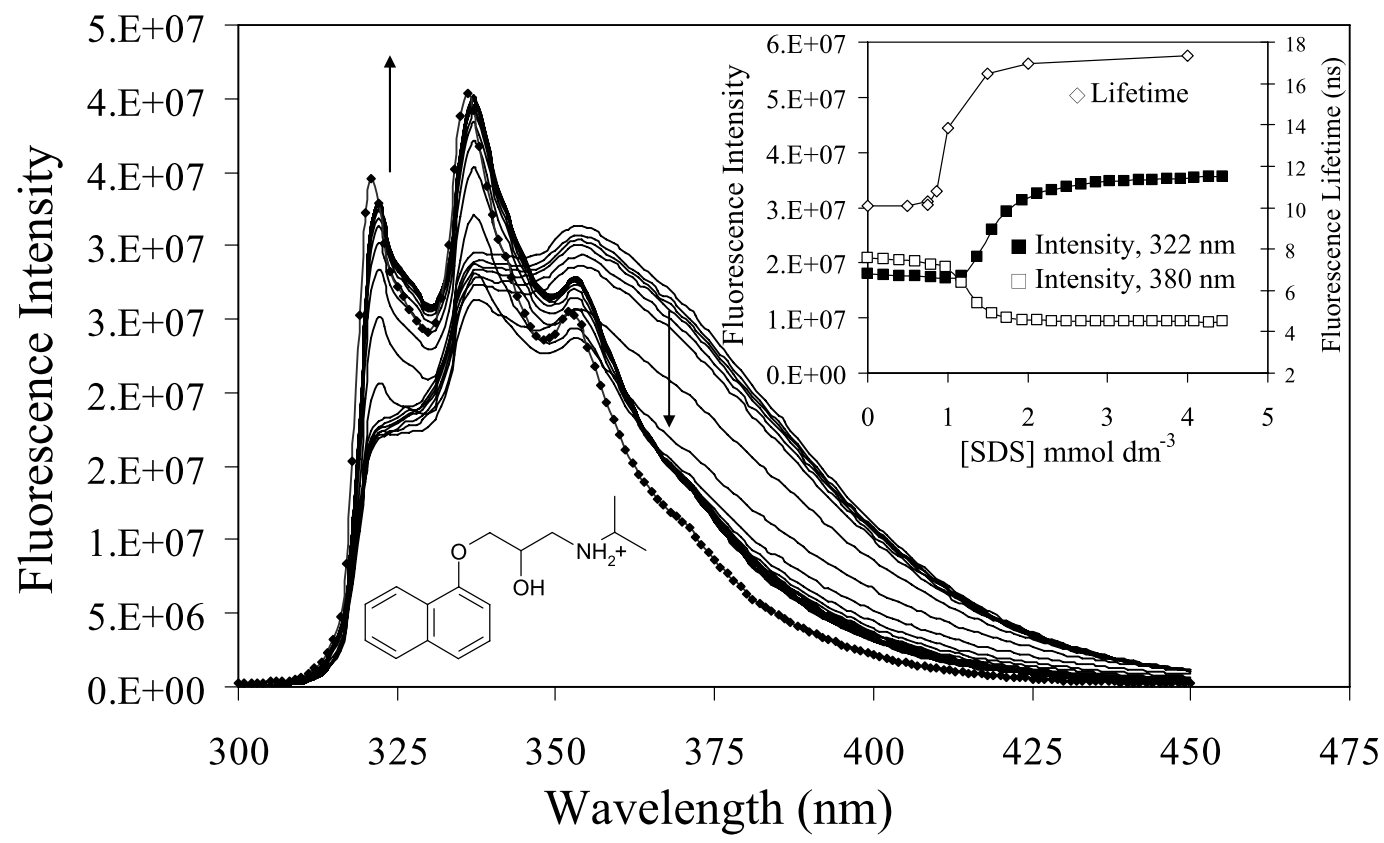

Fig. 1. Fluorescence spectra recorded on increasing SDS concentration $\left(0-4.5 \mathrm{mmol} \mathrm{dm}{ }^{-3}\right)$ in a solution of propranolol $\left(10 \mu \mathrm{mol} \mathrm{dm}^{-3}\right)$ in phosphate buffer $\left(0.1 \mathrm{~mol} \mathrm{dm}^{-3}\right.$ at pH 7.0). The spectrum of propranolol in ethanol $(--)$ is also shown for comparison. INSET: Changes in fluorescence intensity at $322 \mathrm{~nm}\left(\boldsymbol{\square}_{-} \mathbf{-}\right)$ and $380 \mathrm{~nm}(\square-\square)$, and average fluorescence lifetime $(\diamond-\diamond)$ on titration of propranolol with SDS.

was the shorter lifetime of $2.23 \mathrm{~ns}$. In solvents showing the charge transfer band at $\sim 380 \mathrm{~nm}$, the lifetimes and pre-exponential weightings were independent of emission wavelength (325-400 nm) showing that equilibrium between emitting states occurs faster than fluorescence decay. Differences in fluorescence lifetimes between air- and nitrogen-saturated solutions were consistent with diffusion controlled quenching by $\mathrm{O}_{2}\left(k \approx 2 \times 10^{10} \mathrm{dm}^{3} \mathrm{~mol}^{-1} \mathrm{~s}^{-1}\right.$ in ethanol). In SDS solutions the lifetime became biexponential with a dominant (96\%) longer lifetime of $17.7 \mathrm{~ns}$ and the average lifetime increased abruptly at the CMC (inset to Fig. 1).

\section{Fluorescence quenching}

Fluorescence quenching by iodide was used to determine the solvent accessibility of propranolol when associated with SDS micelles and $\beta$-cyclodextrin. Figure 2 shows the Stern-Volmer plots for quenching of fluorescence intensities by iodide anion. In solutions of propranolol alone these show a slight upwards curvature indicative of a major dynamic quenching component $\left(K_{\mathrm{D}}\right)$ with a minor static component $\left(K_{\mathrm{S}}\right)$. Evaluation as outlined by Lakowicz [13] gave a value of $K_{\mathrm{D}}=71 \mathrm{dm}^{3} \mathrm{~mol}^{-1}$. Combined with the fluorescence lifetime of $10.0 \mathrm{~ns}$, this gives a second-order rate constant for dynamic quenching of $7.1 \times 10^{9} \mathrm{dm}^{3} \mathrm{~mol}^{-1} \mathrm{~s}^{-1}$. In the presence of SDS concentrations above the CMC, the overall SternVolmer quenching constant, $K_{\mathrm{SV}}$, reduces to $1.3 \mathrm{dm}^{3} \mathrm{~mol}^{-1}$. This is consistent with the location of the naphthalene ring of propranolol within the micellar structure that is inaccessible to iodide in the aqueous compartment. 


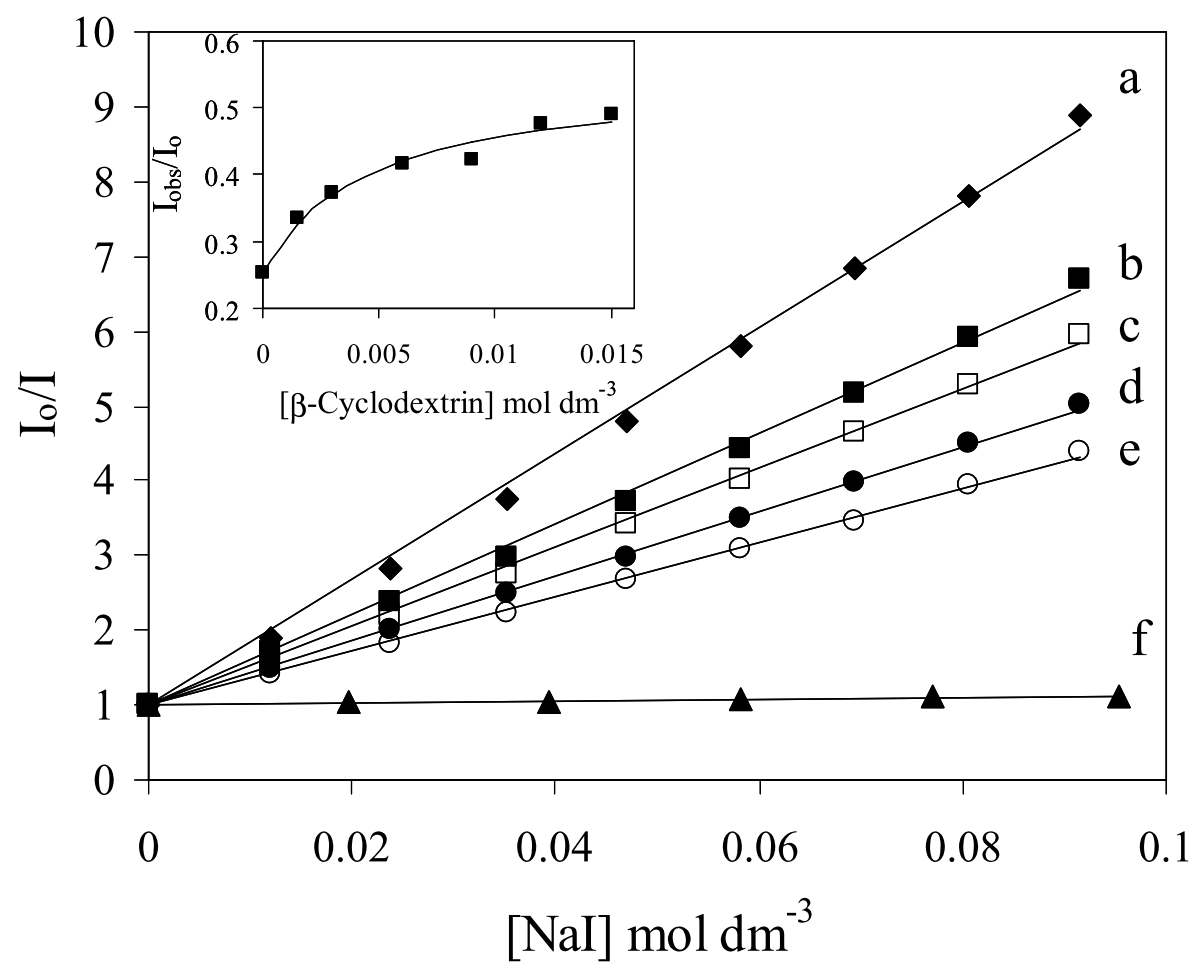

Fig. 2. Stern-Volmer plots for quenching of propranolol by iodide in aqueous solution buffered to $\mathrm{pH} 7$ with phosphate $\left(0.1 \mathrm{~mol} \mathrm{dm}^{-3}\right)$. Solutions contained: (a) propranolol only; (b) $1.5 \mathrm{mmol} \mathrm{dm}{ }^{-3} \beta$-cyclodextrin; (c) $3 \mathrm{mmol} \mathrm{dm}^{-3}$ $\beta$-cyclodextrin; (d) $6 \mathrm{mmol} \mathrm{dm}{ }^{-3} \beta$-cyclodextrin; (e) $12 \mathrm{mmol} \mathrm{dm}^{-3} \beta$-cyclodextrin; (f) $4 \mathrm{mmol} \mathrm{dm}^{-3}$ SDS. INSET: Plot of Eq. (1) (see text) for a solution of propranolol $\left(10 \mu \mathrm{mol} \mathrm{dm}{ }^{-3}\right)$ containing $\mathrm{NaI}\left(35 \mathrm{mmol} \mathrm{dm}^{-3}\right)$.

A previous investigation of the association between propranolol and $\beta$-cyclodextrin by fluorescence spectroscopy [9] showed that there was $<10 \%$ increase in propranolol fluorescence on complex formation. This was too small for accurate evaluation of the association constant. The binding of propranolol by $\beta$-cyclodextrin is readily detected (Fig. 2) by a decrease in quenching of propranolol fluorescence by iodide. The observed fluorescence intensity $\left(I_{\mathrm{obs}}\right)$ as a function of $\beta$-cyclodextrin and iodide concentrations yields values of the Stern-Volmer constants, $K_{\mathrm{f}}, K_{\mathrm{b}}$, for diffusional quenching of propranolol free in solution and bound to cyclodextrin respectively, together with the association constant for propranolol and $\beta$-cyclodextrin, $K_{\mathrm{A}}$, as described by Eq. (1), where $I_{\mathrm{o}}$ is the intensity in the absence of iodide and $\beta$-cyclodextrin:

$$
\frac{I_{\mathrm{obs}}}{I_{\mathrm{o}}}=\left[\frac{1}{1+K_{\mathrm{A}}[\beta-C D]}\right]\left[\frac{1}{1+K_{\mathrm{f}}[Q]}+\frac{K_{\mathrm{A}}[\beta-C D]}{1+K_{\mathrm{b}}[Q]}\right] .
$$

Figure 2 shows the effect of adding $\beta$-cyclodextrin to solutions of propranolol and iodide and nonlinear fitting gives a value of $K_{\mathrm{A}} \approx 195 \mathrm{dm}^{3} \mathrm{~mol}^{-1}$, in good agreement with a value of $239 \mathrm{dm}^{3} \mathrm{~mol}^{-1}$ from calorimetry [8]. The fitted values of $K_{\mathrm{f}}$ and $K_{\mathrm{b}}$ were 80.7 and $23.3 \mathrm{dm}^{3} \mathrm{~mol}^{-1}$, respectively, and the second-order rate constant for quenching by iodide of bound propranolol was $2.3 \times 10^{9} \mathrm{dm}^{3} \mathrm{~mol}^{-1} \mathrm{~s}^{-1}$. $K_{\mathrm{A}}$ for propranolol is similar to that for related molecules such as tryptamine $\left(160 \mathrm{dm}^{3} \mathrm{~mol}^{-1}\right.$ [16]) and serotonin $\left(\sim 53 \mathrm{dm}^{3} \mathrm{~mol}^{-1}[2]\right)$. 
A Stern-Volmer plot (not shown) of propranolol fluorescence lifetimes (determined using two-photon excitation at $630 \mathrm{~nm}$ [6]) shows that dynamic self-quenching occurs with a second-order rate constant of $2.5 \times 10^{9} \mathrm{dm}^{3} \mathrm{~mol}^{-1} \mathrm{~s}^{-1}$. In cells propranolol accumulates to concentrations appreciably greater than millimolar $[3,4]$ and intracellular lifetimes may therefore be shortened by this route.

\section{Multiphoton fluorescence lifetime imaging in mammalian cells}

Fluorescence lifetime images of propranolol uptake by cells $[3,4]$ indicate rapid accumulation to concentrations up to $\sim 10-20 \mathrm{mmol} \mathrm{dm}{ }^{-3}$ within the cell cytoplasm and exclusion from the cell nucleus. The lifetime images and distributions show shorter lifetimes than in solution and are shorter than expected on the basis of self-quenching alone. The observation that the lifetime is shorter than that in water, compared with the longer lifetimes in the less polar systems described above, suggests that intracellular propranolol remains in the cell aqueous compartment rather than being solubilised in lipid environments.

\section{Acknowledgement}

This work was supported by STFC Biomed Network and the University of Salford.

\section{References}

[1] C. Barzanti, R. Evans, J. Fouquet, L. Gouzin, N.M. Howarth, G. Kean, E. Levet, D. Wang, E. Wayemberg, A.A. Yeboah and A. Kraft, Potentiometric determination of octanol-water and liposome-water partition coefficients $(\log P)$ of ionisable organic compounds, Tetrahedron Letters 48 (2007), 3337-3341.

[2] R.H. Bisby, S.W. Botchway, S. Dad and A.W. Parker, Single- and multi-photon excited fluorescence from serotonin complexed with $\beta$-cyclodextrin, Photochemical and Photobiological Sciences 5 (2006), 122-125.

[3] R.H. Bisby, A.G. Crisostomo, S.W. Botchway and A.W. Parker, Fluorescence lifetime imaging of serotonin and other intracellular molecules, in: Central Laser Facility Annual Report, RAL-TR-2007-025, 2006/2007, pp. 101-102; ISBN 9780955661617.

[4] R.H. Bisby, A.G. Crisostomo, S.W. Botchway, A.W. Parker and S.W. Watts, Intracellular distribution of serotonin and propranolol in rat aorta cells, in: Central Laser Facility Annual Report, 2008/2009, pp. 147-148.

[5] R.A. Bissell, A.J. Bryan, A.P. de Silva and C.P. McCoy, Fluorescent PET sensors with targeting/anchoring modules as molecular versions of submarine periscopes for mapping membrane-bound protons, Journal of the Chemical Society, Chemical Communications (1994), 405-407.

[6] S.W. Botchway, A.W. Parker, R.H. Bisby and A.G. Crisostomo, Real-time cellular uptake of serotonin using fluorescence lifetime imaging with two-photon excitation, Microscopy Research and Technique 71 (2008), 267-273.

[7] M.R. Bristow, $\beta$-Adrenergic receptor blockade in chronic heart failure, Circulation 101 (2000), 558-569.

[8] G. Castronuovo and M. Niccoli, Thermodynamics of inclusion complexes of natural and modified cyclodextrins with propranolol in aqueous solution at $298 \mathrm{~K}$, Bioorganic and Medicinal Chemistry 14 (2006), 3883-3887.

[9] A.Y. Glenn, C.A. Fortier, I.V. Jack, X.F. Zhu and I.M. Warner, Chiral recognition of propranolol with $\beta$-cyclodextrin in the presence of 1-and 2-butanol, Journal of Inclusion Phenomena and Macrocyclic Chemistry 51 (2005), 87-91.

[10] D. Hallifax and J.B. Houston, Uptake and intracellular binding of lipophilic amine drugs by isolated rat hepatocytes and implications for prediction of in vivo metabolic clearance, Drug Metabolism and Disposition 34 (2006), 1829-1836.

[11] C.E. Hunt and R.J. Ansell, Use of fluorescence shift and fluorescence anisotropy to evaluate the re-binding of template to (S)-propranolol imprinted polymers, Analyst 131 (2006), 678-683.

[12] J. Kornischka, J. Cordes and M.W. Agelink, 40 Years beta-adrenoceptor blockers in psychiatry, Fortschritte der Neurologie Psychiatrie 75 (2007), 199-210.

[13] J.R. Lakowitz, Principles of Fluorescence Spectroscopy, 2nd edn, Kluwer Academic, New York, 1999, Chapter 8.

[14] A.K. Mukhopadhyay and S. Georghiou, Solvent-induced enhancement of weakly allowed vibronic transitions of aromatic hydrocarbons, Photochemistry and Photobiology 31 (1980), 407-411. 
[15] A. Nakajima, Variations in vibrational structures of fluorescence spectra of naphthalene and pyrene in water and aqueous surfactant solutions, Bulletin of the Chemical Society of Japan 50 (1977), 2473-2474.

[16] A. Orstan and J.B.A. Ross, Investigation of the $\beta$-cyclodextrin-indole inclusion complex by absorption and fluorescence spectroscopies, Journal of Physical Chemistry 91 (1987), 2739-2745.

[17] Siqintuya, Y. Sueishi and S. Yamamoto, Solvent-dependent intramolecular exciplex formation of N-benzyl-Nmethylamine and N,N-dibenzylamine, Journal of Photochemistry and Photobiology A Chemistry 186 (2007), 41-46.

[18] W.K. Surewicz and W. Leyko, Interaction of propranolol with model phospholipids membranes, Biochimica et Biophysica Acta 643 (1981), 387-397. 


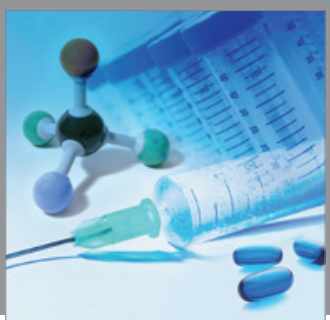

International Journal of

Medicinal Chemistry

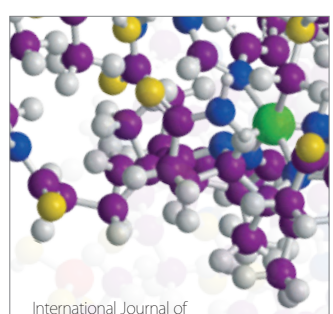

Carbohydrate Chemistry

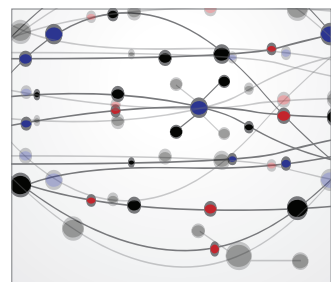

The Scientific World Journal
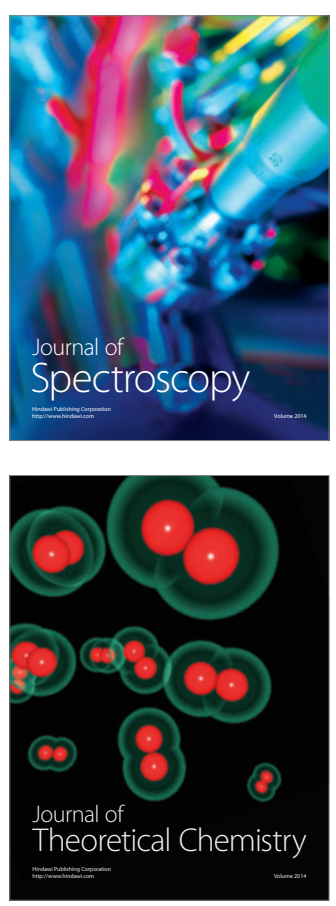
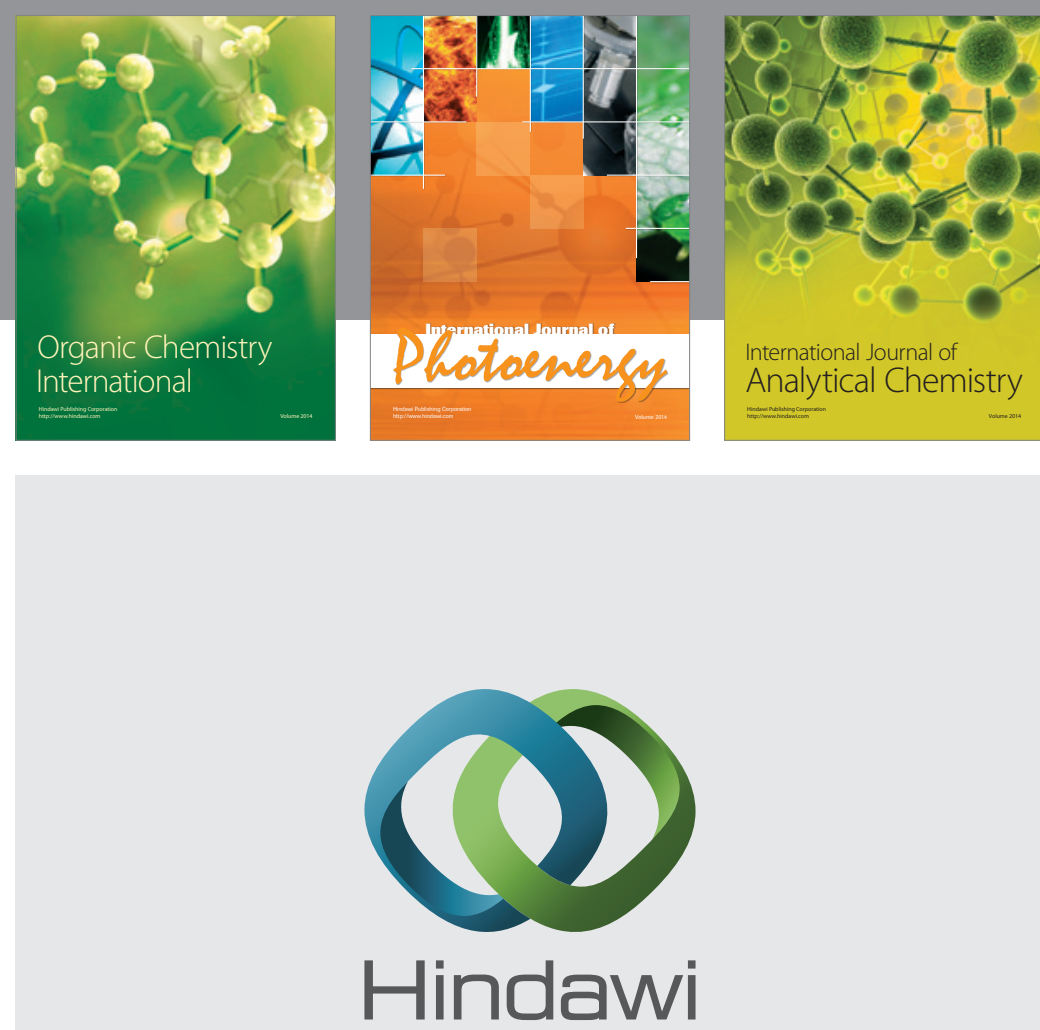

Submit your manuscripts at

http://www.hindawi.com
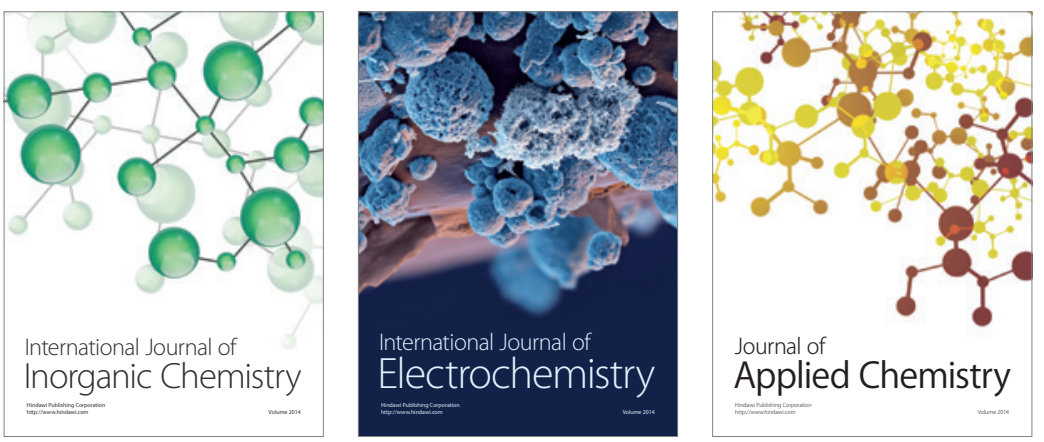

Journal of

Applied Chemistry
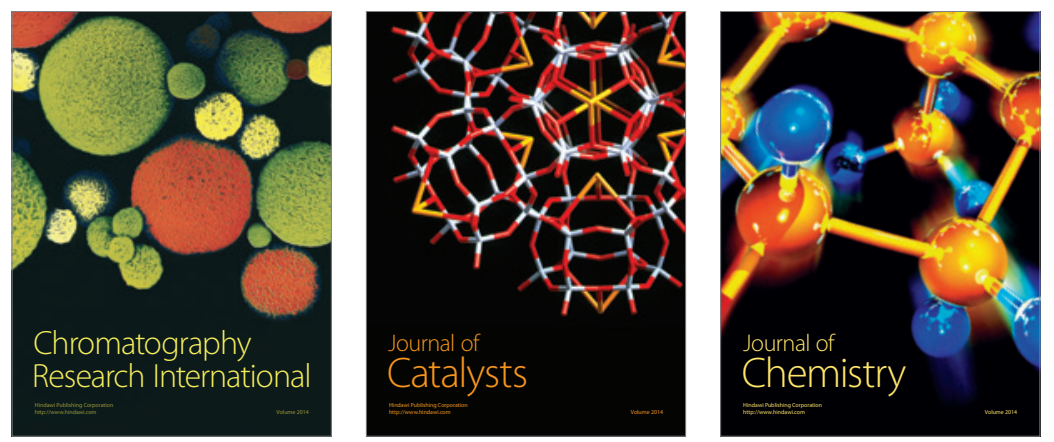
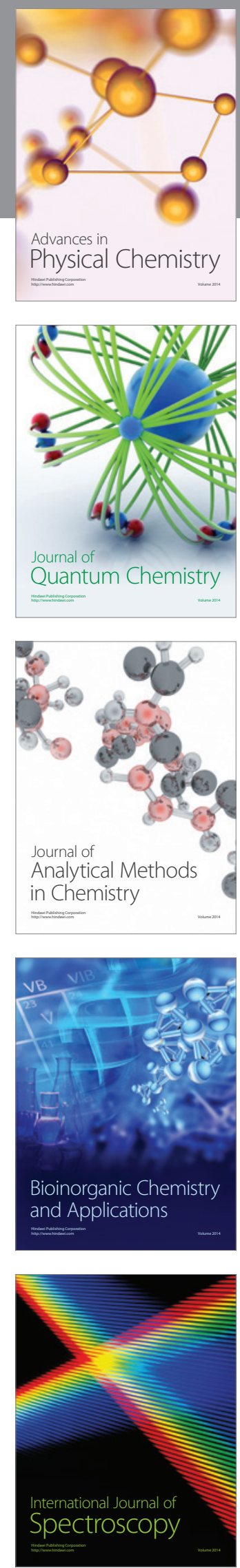